\title{
Impact of coronavirus 2019 (COVID-19) on training and well-being in subspecialty surgery: A national survey of cardiothoracic trainees in the United Kingdom
}

\author{
Edward J. Caruana, MD, MRCSEd, MFSTEd, ${ }^{\mathrm{a}, \mathrm{b}}$ Akshay Patel, MA, MRCS(Eng),
} Simon Kendall, MS, FRCS(CTh), and Sridhar Rathinam, MBBS, FRCS(CTh) ${ }^{\mathrm{a}}$

\section{ABSTRACT}

Objectives: The coronavirus 2019 (COVID-19) pandemic has overwhelmed health care systems and disrupted routine care internationally. Health care workers face disruption to their work routines and professional development, as well as an elevated risk of infection and morbidity. We sought to establish the impact of the COVID-19 pandemic on the well-being, practice, and progression of all trainees in cardiothoracic surgery in the United Kingdom.

Methods: A 31-item questionnaire was designed, validated, and disseminated via email and an instant-messaging platform.

Results: In total, 76 (of 118, 64\%) cardiothoracic surgical trainees responded, representing all training grades and programs nationally; $48(63 \%)$ and $24(32 \%)$ were concerned about their physical and mental health, respectively, $25(33 \%)$ had taken time off work due to COVID-19, 65 (86\%) had treated patients with COVID-19, 36 of whom ( $55 \%$ ) were wearing satisfactory personal protective equipment at the time, $41(54 \%)$ remain concerned about personal protective equipment provision at their institution, $42(55 \%)$ had been redeployed to cover other specialties, and $23(30 \%)$ had encountered ethical dilemmas related to care of patients. There was a significant impact on time spent in outpatient clinics ( $44 \%$ reduction), multidisciplinary team meetings ( $79 \%$ reduction), and operating theaters $(78 \%$ reduction). In total, $67(88 \%)$ of respondents were concerned about the impact on their training, and $54(71 \%)$ felt that the deviation may require an extension in their planned training time.

Conclusions: The duration and impact of the current pandemic is, as yet, uncertain. Timely sharing of experiences, concerns, and expectations will inform health care and education policy and influence practice in the pandemic era and beyond. ( $\mathrm{J}$ Thorac Cardiovasc Surg 2020;160:980-7)

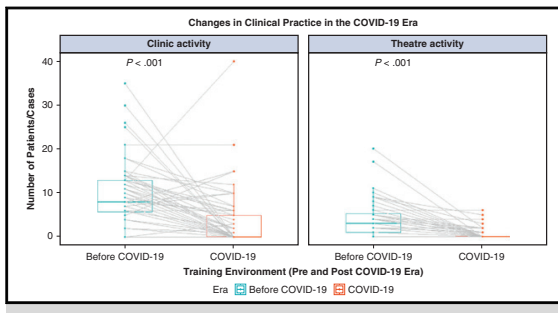

Composite pairwise illustration showing a significant decrease in outpatient clinic and theater case numbers.

\section{CENTRAL MESSAGE}

The COVID-19 pandemic poses significant personal and professional challenges to trainees. Nonetheless, it is a teachable moment_focusing on core values, professionalism, quality, and safety of care.

\section{PERSPECTIVE \\ The experiences and concerns reported in our article reflect findings from national trainees in Cardiothoracic Surgery in the United Kingdom. The themes identified are likely generalizable to other specialties and internationally. They may contribute to determining thoughtful health pol- icy in an ongoing manner as the COVID-19 pandemic enters subsequent phases. \\ See Commentaries on pages 988 and 989.}

From the a Department of Thoracic Surgery, Glenfield Hospital, University Hospitals of Leicester, Leicester; ' ${ }^{\mathrm{b}}$ NIHR Biomedical Research Centre, University of Nottingham, Nottingham; ${ }^{\mathrm{C}}$ Institute of Immunology and Immunotherapy, University of Birmingham, Birmingham; and ${ }^{\mathrm{d}}$ Department of Cardiothoracic Surgery, James Cook University Hospital, Middlesborough, United Kingdom.

Received for publication May 8, 2020; revisions received May 14, 2020; accepted for publication May 21, 2020; available ahead of print June 1, 2020.

Address for reprints: Edward J. Caruana, MD, MRCSEd, MFSTEd, Department of Thoracic Surgery, Glenfield Hospital, Groby Rd, Leicester LE3 9QP United Kingdom (E-mail: edwardcaruana@nhs.net).

$0022-5223 / \$ 36.00$

Copyright (c) 2020 by The American Association for Thoracic Surgery

https://doi.org/10.1016/j.jtcvs.2020.05.052
The emergence of the novel severe acute respiratory syndrome coronavirus 2 has resulted in a global pandemic. ${ }^{1}$ Attempts to meet the demands of caring for patients with coronavirus 2019 (COVID-19) have overwhelmed health care systems internationally. ${ }^{2}$ Clinicians at all career stages

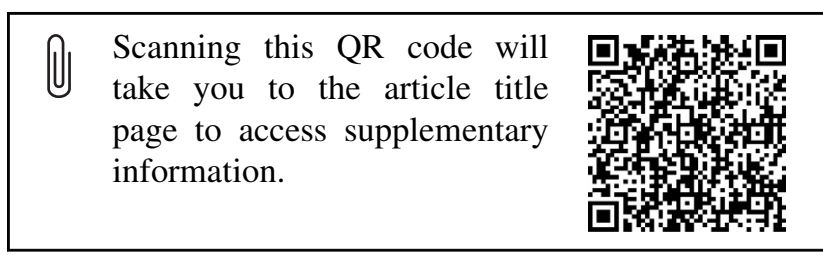




\section{Abbreviations and Acronyms \\ COVID-19 = coronavirus 2019 \\ PPE = personal protective equipment \\ SCTS $=$ Society for Cardiothoracic Surgery in Great Britain and Ireland}

have had their clinical routines disrupted or are being redeployed away from their professional specialty areas to provide care to patients with COVID-19. There is, resultantly, widespread disruption of standard care that is likely to last for many months.

Health care workers are at an increased risk of contracting the virus, and a high rate of morbidity has been noted in countries with a high burden of disease..$^{2-4}$ Trainees form a significant proportion of the medical workforce that has been redeployed to reinforce care provision at the COVID-19 frontline, raising concern that their physical health and mental well-being may be at risk. ${ }^{3}$

This reallocation away from their parent specialty poses numerous challenges, not the least of which being trainees' level of competence and confidence to deliver their new role, particularly in an overstretched system. ${ }^{5}$ It additionally deviates from traditional training pathways and exposures, and it has the potential to negatively impact trainees' professional development in (at least) the short to medium term. The impact of reduced patient throughput and workforce redeployment on training may be even more significant in surgical specialties, wherein consistent hands-on practice is required for the development and maintenance of technical skills. Nonetheless, this situation poses unique learning opportunities across clinical, academic, and leadership domains and may prompt a review of our educational norms. ${ }^{6}$

Furthermore, trainee cardiothoracic surgeons working in a model of socialized medicine (the National Health Service) are, first and foremost, doctors who embrace as privilege the opportunity to contribute to the health of the nation, community, and individual, to the best of their ability, and especially in times of crisis.

We performed a national survey of cardiothoracic surgical trainees in the United Kingdom 3 weeks after the implementation of national lockdown procedures, to assess the impact of the COVID-19 pandemic on their well-being, daily practice, training exposures, and related concerns. To the best of our knowledge, this is the first paper to report on a comprehensive, multifaceted evaluation of trainees' experiences during this pandemic.

\section{METHODS}

\section{Training Structure and Terminology}

Cardiothoracic surgeons in the United Kingdom currently train for at least 10 years after achieving a primary medical qualification, with entry through a national selection process. The specialist training program is of 6 to 8 years' duration, depending on the stage of appointment. Trainees initially achieve competence in the generality of surgery and the range of cardiothoracic practices and subsequently hone their training in the latter years to 1 of 2 specialist areas: adult cardiac surgery or general thoracic surgery. Subspecialist practice, including in congenital cardiothoracic surgery and transplant surgery, requires further exposure through fellowships.

The European Working Time Directive limits working patterns to an average 48-hour week, including for surgeons in training. Daytime working is often considered in blocks of activity, or "sessions," defined as 4-hour (or half-day) blocks. Trainees also work antisocial hours, that is during night time, weekends, and public holidays. Structured learning is facilitated nationally by means of a comprehensive portfolio of courses $^{8}$ and supplemented regionally through didactic and simulationbased teaching programs. The Society for Cardiothoracic Surgery in Great Britain and Ireland (SCTS) also offers a portfolio of traveling fellowships to enable trainees consolidate their clinical expertise before consultant appointment.

Trainees in the specialty are expected to have performed a minimum of 250 major cases by the time of final certification. ${ }^{9}$ There is an online portfolio (www.iscp.ac.uk) of learning, designed to record progression across various domains. This documentation is facilitated by means of structured forms known as "Workplace-Based Assessments" that require input and validation from trainees and trainers. The Specialty Advisor Committee for Cardiothoracic Surgery has established a minimum requirement for 50 of these forms to be completed in a given training year and for these to span the breadth of the training curriculum. ${ }^{10}$ There is additionally a requirement for evidence of learning and engagement in research, quality improvement work, medical education, management, and leadership.

\section{Survey Design and Dissemination}

A 31-item questionnaire (Online Data Supplement) was designed by one of the authors (E.J.C.), evaluated for face, content, and construct validity (A.P., S.R.), and pretested by 5 cardiothoracic surgical colleagues across trainee and consultant grades.

The survey was developed on an online platform (onlinesurveys.ac.uk) and circulated to all nationally appointed trainees in cardiothoracic surgery in the United Kingdom for whom contact details were available, via e-mail and group instant messaging (WhatsApp), with a reminder at 48 hours. It was kept open between Sunday, April 12, 2020, and Wednesday, April 15 , 2020. Submission of partially completed questionnaires was not permitted. No financial or other incentives were provided for participation. Formal ethics approval was not required for this work carried out in conjunction with national stakeholder bodies: The Royal College of Surgeons of Edinburgh and the SCTS.

\section{Reporting and Statistical Analysis}

For the purposes of data analysis, "early-stage" trainees were defined as those in the first 6 years of training, whereas "late stage" referred to colleagues in their final or penultimate training years. Trainees whose grade could not be accurately identified, including those in full-time research, were excluded from this subgroup analysis. Time period comparisons were made between routine practice "pre-COVID era" and current practice "COVID era," defined by individual respondents according to the date in which a shift in practice was noted at their institution or region.

For continuous variables, results are expressed as means and standard deviations and for categorical variables as counts and percentages. The denominator for percentages is the full respondent cohort $(n=76)$ unless otherwise specified.

For continuous data, intergroup comparison was performed using the Student $t$ test or Mann-Whitney $U$ test/pairwise Wilcoxon rank sums test, depending on the distribution of data. Differences between groups for categorical data were assessed using the Fisher exact test of independence. Univariate analyses of clinical activities between different time 
periods were assessed by performing individual unadjusted logistic regression analysis with inclusion of one covariate per model. Missing data were excluded from analyses. Correction for multiple comparisons was applied using the Benjamini-Hochberg method.

All analyses were performed using the R 3.6.2 program via RStudio, Inc for Windows, R Core Team (2013; R Foundation for Statistical Computing, Vienna, Austria) by 2 authors (A.P., E.J.C.). ${ }^{11}$

\section{RESULTS}

In total, $76(64 \%)$ of the 118 nationally appointed trainees in cardiothoracic surgery in the United Kingdom responded, representing all training grades and each of the 14 training regions in the United Kingdom and the Republic of Ireland.

Of these, immediately before the onset of the pandemic, $53 \%(\mathrm{n}=40)$ were working in adult cardiac surgery, $28 \%$ $(n=21)$ in general thoracic surgery, $5 \%(n=4)$ in mixed cardiothoracic practice, and the remaining $13 \%(\mathrm{n}=10)$ in other specialties.

\section{COVID-19 Illness, Exposure, and Personal Protection}

The majority of trainees reported being at least somewhat concerned about their physical $(63 \%, \mathrm{n}=48)$ and mental $(32 \%, n=24)$ well-being. In total, $61 \%(n=46)$ of trainees felt that they would not have access to COVID19 testing for themselves or their families, should they come to need it.

A total of $33 \%(n=25)$ of all trainees reported having taken time off work due to COVID-19-related issues, with a median time off work of 7 days (range, 2-18); $9 \%$ $(\mathrm{n}=7)$ reported isolating due to a suspected or confirmed case in their household, $4 \%(n=3)$ identified as vulnerable or high risk, and $20 \%(\mathrm{n}=15)$ were symptomatic. Of those with symptoms, $11 \%(\mathrm{n}=8)$ were never tested for COVID$19,4 \%(n=3)$ tested negative, and 5\% $(n=4)$ tested positive.

A significant proportion of trainees felt they had not received suitable training on personal protection when treating patients with COVID-19 $(24 \%, \mathrm{n}=18)$, with $30 \%$ $(\mathrm{n}=23$ ) having not been fitted or tested on appropriate face masks at the time of responding to the survey. In addition, 54\% $(n=41)$ expressed concerns about the availability of appropriate personal protective equipment (PPE) in their clinical areas. This, despite $86 \%(n=65)$ having had clinical contact with positive or suspected patients with COVID-19 and 33\% $(n=25)$ having operated on this patient group. Only 55\% $(n=36 / 65)$ of those with known clinical contact reported having been wearing satisfactory levels of PPE during these encounters.

\section{Clinical Practice}

The majority of trainees $(55 \%, \mathrm{n}=42)$ reported a change in their working hours, with the most common being an increase in antisocial hours $(52 \%, \mathrm{n}=22 / 42)$, greater overall working hours $(45 \%, \mathrm{n}=19 / 42)$, and a switch to a full on-site (from off-site on-call) working pattern $(33 \%, \mathrm{n}=14 / 42)$.

At the time of reporting, $45 \%(n=34)$ of trainees were continuing to work exclusively in their original specialty area, whereas $34 \%(n=26)$ were spending less than one half their working hours caring for patients in their original specialty. There was no difference in the likelihood of being redeployed between early or late stages of training (Table 1).

Of the 42 trainees who were redeployed, $38 \%(n=16)$ were newly covering both cardiac and thoracic surgery, $64 \%(\mathrm{n}=27)$ were working in critical care, and $52 \%$ $(\mathrm{n}=22)$ were covering other surgical or medical specialties at least some of the time. Only 5\% $(n=4)$ reported not feeling confident in their role.

In total, $30 \%(n=23)$ have experienced ethical dilemmas related to futility or refusal of care that directly affected their patients. These were related to both routine surgical patients and patients with COVID-19, and broadly fell under the themes of (1) delivering suboptimal care alternatives to patients who would routinely receive surgery with curative intent or prognostic benefit, (2) operating on highrisk surgical patients without suitable intensive care backup, and (3) restricting care delivery to preserve resources for a potential increase in the number of patients requiring support for COVID-19-related illnesses.

Trainees reported a significant deviation in their sessional activity across a variety of clinical activities (summarized in Table 2), including outpatient clinics $(P<.0001)$,

TABLE 1. Impact of early versus late stage of training on likelihood of being redeployed to another specialty, being engaged in COVID-19-specific research, reporting valuable learning in current role during COVID-19 era, and the possibility of requiring an extension to planned training time (a deferral of completion of training)

\begin{tabular}{|c|c|c|c|c|}
\hline & Early stage $(\mathrm{n}=\mathbf{5 3})$ & Late stage $(n=19)$ & Odds ratio $(95 \% \mathrm{CI})$ & $P$ value \\
\hline Redeployed & $29(54.7 \%)$ & $11(57.9 \%)$ & $1.14(0.35-3.83)$ & 1.000 \\
\hline Engaged in COVID-19 research & $21(39.6 \%)$ & $5(26.3 \%)$ & $0.55(0.134-1.93)$ & .406 \\
\hline Valuable learning in current role & $24(45.3 \%)$ & $7(36.8 \%)$ & $0.71(0.20-2.32)$ & .596 \\
\hline May require extended training time & $36(67.9 \%)$ & $14(73.7 \%)$ & $1.32(0.38-5.45)$ & .775 \\
\hline
\end{tabular}

CI, Confidence interval; COVID-19, coronavirus 2019. 
TABLE 2. Percentage change in sessional activity between the preCOVID-19 and COVID-19 era

\begin{tabular}{lc}
\hline \multicolumn{1}{c}{ Activity } & Percent deviation, mean \pm SD \\
\hline Outpatient clinic & $-44 \pm 81$ \\
Multidisciplinary team meetings & $-79 \pm 51$ \\
Inpatient/ward care & $+68 \pm 143$ \\
Operating theater & $-78 \pm 31$ \\
Research and audit & $+3 \pm 94$ \\
Structured learning & $+51 \pm 172$ \\
Overall & $-13 \pm 95$ \\
\hline
\end{tabular}

One session is half a day or 4 hours. A negative (-) deviation signifies less time spent on that activity during the COVID-19 era. $S D$, Standard deviation.

multidisciplinary team meetings $(P<.0001)$, inpatient/ ward care $(P=.012)$, operating theaters $(P<.0001)$, and structured learning sessions $(P<.0001)$. There was no difference in the time spent on research and clinical audit activity $(P=.82)$.

\section{COVID-19 Research}

The reported engagement with and contribution to COVID-19-specific academic work was highly variable, with $66 \%(\mathrm{n}=50)$ currently having no participation, but with $76 \%$ of these colleagues $(n=38 / 50)$ expressing an interest in developing this role. Of those who reported being research- or audit-active in this field, $77 \%(\mathrm{n}=20 / 26)$ are primarily engaged in national or international collaborative initiatives. There was no difference noted between early- and late-stage trainees' engagement with COVID19 research (Table 1$)$.

\section{Training and Progression}

The majority of trainees report being somewhat $(25 \%, \mathrm{n}=19)$ or very $(63 \%, \mathrm{n}=48)$ concerned about the impact of the pandemic on their learning and progression through structured training. There was no association between trainee seniority and declared concern (Table 1).

Most $(57 \%, \mathrm{n}=43)$ feel that their current role offers little to no relevant learning opportunities and is in fact a deterrent to their professional development. Only $14 \%$ $(\mathrm{n}=11)$ report a comparable or above-average-value learning experience. There is also very poor documentation of learning encounters in the current environment, with only $9 \%(\mathrm{n}=7)$ of trainees having completed Workplace-Based Assessments, and $65 \%(n=49)$ reporting that to do so

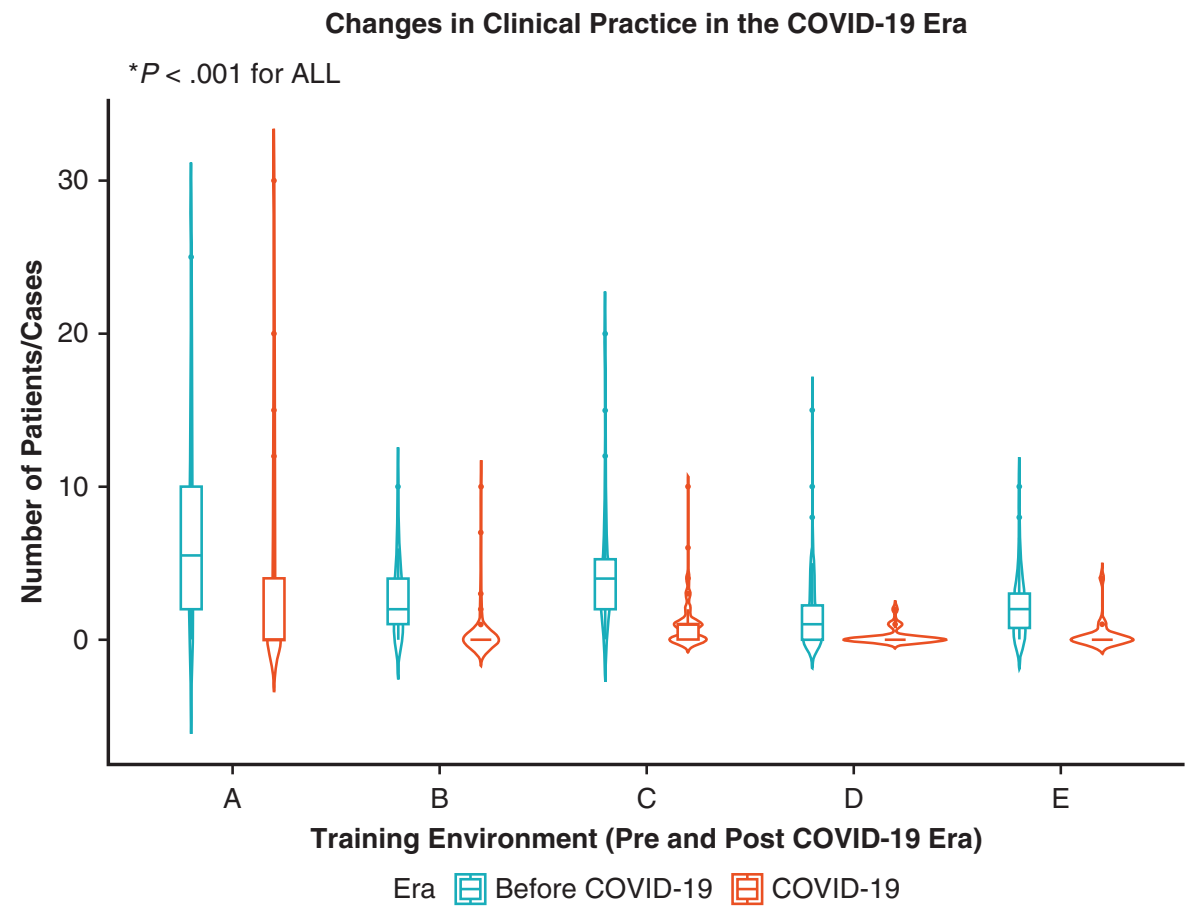

FIGURE 1. Violin plots showing comparisons of the number of (A) follow-up patients reviewed in outpatient clinic $(P<.001)$, (B) new patients reviewed in outpatient clinic $(P<.001),(\mathrm{C})$ cases assisted in theater $(P<.001)$, (D) minor cases performed as primary surgeon $(P<.001)$, and $(\mathrm{E})$ major cases performed by individual trainees $(P<.001)$, in the pre-COVID-19 and COVID-19 eras. The plots indicate significant drops in levels of clinical activity across all environments because of the COVID-19 pandemic. Frequency of individual case or patient numbers is shown by the width of the individual violin plots. Statistical significance calculated using the pairwise Wilcoxon rank sum method. COVID-19, Coronavirus 2019. 


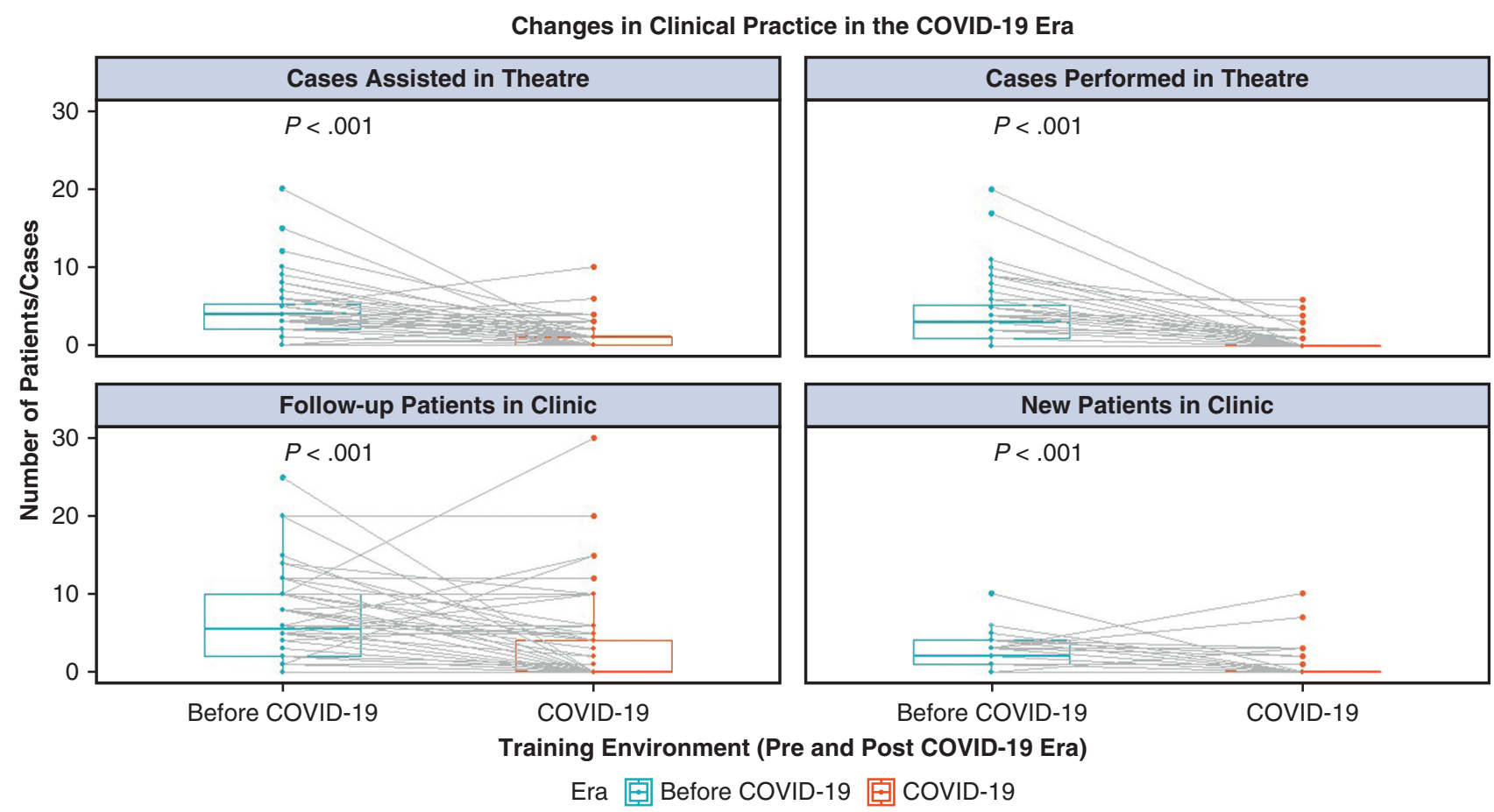

FIGURE 2. Paired comparison of the number of individual trainee's declared patient consultations in clinic, and procedures assisted or performed in theater, between routine practice and the COVID-19 era. Pairwise demonstration of a significant drop in clinical activity by caseload and patient number in 2 different clinical environments because of the COVID-19 pandemic. Statistical significance calculated using the pairwise Wilcoxon rank sum method. COVID-19, Coronavirus 2019.

would neither be achievable nor appropriate to attempt in the current climate.

Trainees generally reported a reduction in clinical encounters in the outpatient clinic, for both new and followup clinics, as well as a significantly restricted participation in surgical procedures as both an assistant and primary surgeon (Figures 1 and 2). There appears to be a disproportionate decline in entrustment in addition to a reduction in any operative participation, with trainee-reported numbers indicating they would perform $26 \pm 28 \%$ of total departmental cases as primary surgeon routinely but only $13 \pm 26 \%$ in the COVID-19 era.

There was no difference between early- and late-stage trainees in the degree to which an individual trainee's clinic

TABLE 3. Disruption of professional leave and events due to the COVID-19 pandemic

\begin{tabular}{lc}
\hline \multicolumn{1}{c}{ External professional commitment } & n (\%) \\
\hline Study leave for personal study & $41(54)$ \\
Professional examination(s) & $21(28)$ \\
Clinical/academic interview(s) & $6(8)$ \\
Academic conference(s) & $54(71)$ \\
Clinical immersion/fellowship visit(s) & $11(15)$ \\
At least one professional event cancelled & $68(89)$ \\
\hline
\end{tabular}

consultations $(60 \pm 61 \%$ vs $69 \pm 72 \%$ decrease, $P=.616)$ and operative case numbers as primary surgeon $(91 \pm 21 \%$ vs $81 \pm 33 \%$ decrease, $P=.2972$ ) were diminished.

Numerous trainees have had their attendance to a variety of professional development events disrupted by the COVID-19 pandemic (outlined in Table 3). There is a thirst for continuing educational opportunities, with $93 \%$ $(n=65)$ of trainees stating they would engage with a regular online program, and with an overwhelming request for a topic focus on cardiothoracic surgical curriculum items $(97 \%, \mathrm{n}=64)$ as opposed to COVID-19-related topics $(49 \%, \mathrm{n}=32)$.

A significant proportion of trainees $(32 \%, \mathrm{n}=24)$ feel that the current interruption to their training already merits an extension to their planned training time, with the absolute majority $(71 \%, \mathrm{n}=54)$ expressing a preference for this, should the current disruption last beyond 3 months. There was no difference in expressed preference between junior and senior trainees $(P=.96)$.

Similarly, $71 \%(\mathrm{n}=54)$ of respondents are concerned that there is currently insufficient communication and clarity from the training bodies and training leadership, with regards to the impact of the current pandemic on training plans, or that the proposals that have been shared may not be sufficiently specific or deliverable. 
TABLE 4. Trainee recommendations on practice and training amidst the COVID-19 pandemic

Commonly expressed trainee perspectives

Day-to-day practice

Wider access to testing of surgical patients and clinical staff would serve to reassure all parties and restore effective care.

A pragmatic view should be taken to facilitate, support, and promote COVID-19-related research participation and collaboration.

Training progression

Trainee progression should be considered on an individual basis, with flexibility and mutual agreement on duration and purpose of any extension period.

Competency and not clinical training time in the specialty—as evaluated by trainers in discussion with individual trainees—should underpin panel

decisions on progression.

Specific and attainable adaptations to training need to be agreed, mapped and communicated by national training bodies.

Support

Online learning interventions should be made available, focusing on (1) clinical topics relevant to cardiac and thoracic surgery and (2) the conduct and delivery of research.

COVID-19, Coronavirus 2019.

Valuable insight has also been derived from trainees' comments when questioned about their ideas, concerns, and expectations relevant to their training and clinical practice in the COVID-19 era and beyond. These are thematically summarized and presented in Table 4.

\section{DISCUSSION}

The impact of the COVID-19 pandemic on patient outcomes has been widely documented, albeit still poorly quantified. $^{12}$ A significant direct and indirect morbidity and mortality burden is expected to continue for many months.

Health care professionals subsume the risks associated with their profession in daily practice, seeking to act in their patients' best interests. In times of epidemic or pandemic, this risk is amplified, and clinicians suffer a greater prevalence of infection and complications than the baseline population. $^{13}$ The high incidence of health care workers requiring time off work for COVID-19 illness or quarantine is detrimental to both individuals and the institutions at which they work. Social distancing and isolation measures aimed at curtailing transmission have also forced hospitals and departments to take measures to ensure an adequate reserve capacity.

Although risk is inherent in clinical practice, and should not prejudice the care delivered, it is essential to empower staff by providing adequate levels of personal protection. ${ }^{14}$ It is disappointing to note that a majority of our trainees report not having consistent or reliable access to appropriate PPE, and that access to testing for patients and staff remains heavily restricted. Clinicians, including trainees, will continue to be exposed to patients infected with severe acute respiratory syndrome coronavirus 2 for many months to come, across a variety of clinical settings. Health care delivery should be facilitated with the supply of appropriate equipment. Where necessary, thoughtful repurposing of materials initially designed for alternate use may be appropriate, insofar as efficacy and clinician safety are not compromised. ${ }^{15}$

Our work also highlights variations in clinician education with regards to safe and appropriate use of PPE. This is an issue that needs to be actively addressed acutely and in an ongoing manner, as the recommendations and equipment available to health care staff continue to evolve.

Concerns about clinicians' mental well-being are to be expected in the context of a global pandemic, particularly in the context of burdensome challenges in decision making, requiring a deviation from established norms. ${ }^{16}$ It is positive to note that these have been acknowledged by trainees in our survey; however, this mandates a constructive, considered, and proportionate response to support these colleagues in managing their anxieties and ailments.

Various health care institutions have attempted to mitigate concerns of exposure and burnout in different ways, often adopting bloc working patterns that allow distancing from the biological and emotional burdens of clinical practice in times of a pandemic.

Opportunities for surgical training, particularly in theater, have declined in recent years and have been compounded by a restriction on working hours. ${ }^{17,18}$ Evidence of further diminished training opportunities during the current pandemic is already emerging. ${ }^{19}$ with efforts being made in an attempt to identify compensatory interventions to maintain a level of structured learning. ${ }^{6,20}$ Our data corroborate these findings, which appear to impact trainees similarly across training levels. It is concerning, albeit unsurprising, to note that entrustment is decreasing alongside time spent in theater by trainees. Although consultant-delivered care is expected in the initial-phase uncertainties of a pandemic, a new norm must quickly be established wherein training is maximally delivered. Every clinical encounter must be approached and 
embraced as an invaluable learning opportunity in a time of diminished caseload.

Innumerable initiatives have been established in an attempt to mitigate the reduced clinical training opportunities. Measures such as establishing remote supervision with trainers, conferences and lectures being moved to virtual platforms, ${ }^{21}$ continuing support for a variety of forward-looking COVID- and non-COVID-related research initiatives and grant applications, and postponing exit examinations are welcome initiatives, and each play a role in maintaining a culture of continuing education through the pandemic and thereafter. ${ }^{22}$ It is important to recognize that this pandemic has facilitated the emergence of clinical leaders, also from among the trainee body. It has also exposed the potential for efficiently effecting changes in systems previously apparently set in stone.

The sometimes-intangible learning from these unique exposures and contributions must be consolidated by means of individual reflective practices. It would also be timely for educational bodies to consolidate and disseminate emerging best practices in training, so as to allow programs to identify strengths and weaknesses in their adaptations to date and to benefit from the learning experiences of global colleagues.

The Statutory Education Bodies, the Postgraduate Deans, the Joint Committee on Surgical Training, many Specialty Advisory Committees, including that for Cardiothoracic Surgery, and the SCTS have remained in regular communication with trainees and trainers alike, focusing on issues related to well-being, sustainable delivery of training, and concerns for progression. The key recommendations have included (1) the postponement of examinations and courses, with allowances for extension of training for those impacted, (2) introducing flexibility in terms of the evidence that may be considered by review panels, (3) introducing novel progression outcomes that recognize the limitations of surgical training during a pandemic, thus avoiding undue detriment to candidates, ${ }^{23}$ and (4) opting for a delay in launching curriculum changes previously intended for August 2020. During this period of travel restriction, the SCTS has extended the time period for use of fellowship funding, and has streamlined its educational portfolio across Web-based and hybrid simulation platforms.

The Specialty Advisory Committee for Cardiothoracic Surgery has reiterated to trainees its commitment to facilitating and ensuring the quality of training. Discussions are actively ongoing to devise specific, actionable recommendations for establishing a new educational normal that embraces the opportunity and renewed impetus presented by these challenging times.

This work highlights several important perspectives of trainees at a time of dynamic external pressures, with implications for individuals and organizations at a national level and beyond. This semiqualitative study enabled us to provide a "national snapshot" of the early impact of the COVID-19 pandemic on cardiothoracic trainees in the United Kingdom. The Internet-based survey design allowed for comprehensive reach of relevant stakeholders, and detailed information gathering (despite tackling complex and sensitive issues), in a nonthreatening, time-efficient, and cost-effective manner. This is attested by the high response rate, despite the challenging times at which it was delivered. The authors acknowledge the limitations of this work, in that (1) it likely does not fully encompass all aspects of relevance to this multifaceted, unpredictable, and constantly evolving situation; (2) that the data synthesis may be subject to researcher bias; and (3) that the output may omit additional core themes that could emerge over time and with further research. We additionally acknowledge (4) that the survey methodology is susceptible to response bias, despite the option of anonymity.

\section{CONCLUSIONS}

The COVID-19 pandemic poses significant personal and professional challenges to trainees. Nonetheless, this crisis is a teachable moment, one for stakeholders to step up and demonstrate an unyielding focus on core medical values, professionalism, quality, and safety of care. Surgical trainees remain determined to maintain their professional development alongside care delivery to diverse patient groups as determined by clinical need. Educational and professional bodies should embrace the opportunity to critically appraise and challenge establish practices while seeking to dynamically support education and well-being, and to emerge from the pandemic era with unique insight for establishing a new "normal."

\section{Conflict of Interest Statement}

Dr Caruana is trainee representative on the Cardiothoracic Surgical Specialty Board at the Royal College of Surgeons of Edinburgh. Dr Kendall is President of the Society for Cardiothoracic Surgery in Great Britain and Ireland. Dr Rathinam is Education Secretary for the Society for Cardiothoracic Surgery in Great Britain and Ireland, and chairs the Cardiothoracic Surgical Specialty Board at the Royal College of Surgeons of Edinburgh. Dr Patel reported no conflicts of interest.

The Journal policy requires editors and reviewers to disclose conflicts of interest and to decline handling or reviewing manuscripts for which they may have a conflict of interest. The editors and reviewers of this article have no conflicts of interest. 
The authors acknowledge the contribution of Abdul Badran (National Trainee Representative for Cardiothoracic Surgery) in coordinating the circulation of the online survey.

\section{References}

1. World Health Organization. WHO announces COVID-19 outbreak a pandemic; 2020. Available at: http://www.euro.who.int/en/health-topics/health-emergenci es/coronavirus-covid-19/news/news/2020/3/who-announces-covid-19-outbreaka-pandemic. Accessed May 4, 2020.

2. Remuzzi A, Remuzzi G. COVID-19 and Italy: what next? Lancet. 2020;395: 1225-8.

3. Lai J, Ma S, Wang Y, Cai Z, Hu J, Wei N, et al. Factors associated with mental health outcomes among health care workers exposed to coronavirus disease 2019. JAMA Netw Open. 2020;3:e203976.

4. Wang D, Hu B, Hu C, Zhu F, Liu X, Zhang J, et al. Clinical characteristics of 138 hospitalized patients with 2019 novel coronavirus-infected pneumonia in Wuhan, China. JAMA. 2020;323:1061.

5. Plans regarding trainee redeployment during the COVID-19 pandemic. Academy of Medical Royal Colleges; 2020. Available at: https://www.aomrc.org.uk/atdgpapers-reports/plans-regarding-trainee-redeployment-during-the-covid-19-pand emic/. Accessed May 4, 2020

6. Kogan M, Klein SE, Hannon CP, Nolte MT. Orthopaedic education during the COVID-19 pandemic. J Am Acad Orthop Surg. 2020;28:e456-64.

7. Zakkar M, Benedetto U, Angelini GD, Murphy G, Shah R, Jahangiri M, et al. Cardiothoracic surgery training in the United Kingdom. J Thorac Cardiovasc Surg. 2019;157:1948-55.

8. Moorjani N, Lewis M, Shah R, Barnard S, Graham T, Rathinam S. Implementation of a novel portfolio of structured, curriculum-aligned, simulation-based, cardiothoracic surgery training courses: evolving the delivery of surgical education. J Thorac Cardiovasc Surg. 2017;154:2009-16.

9. JCST. Certification guidelines \& checklists; 2020. Available at: https://www.jcst. org/quality-assurance/certification-guidelines-and-checklists/. Accessed May 4, 2020.

10. Cardiothoracic surgery curriculum; 2020. Available at: https://www.gmc-uk.org/ education/standards-guidance-and-curricula/curricula/cardiothoracic-surgerycurriculum. Accessed May 4, 2020.

11. R: The R Project for Statistical Computing. Available at: https://www.r-project. org/. Accessed May 4, 2020
12. Wu Z, McGoogan JM. Characteristics of and important lessons from the coronavirus disease 2019 (COVID-19) outbreak in China. JAMA. 2020; 323:1239.

13. Resolving Ethical Dilemmas: A Guide for Clinicians, 5e. Lippincott, William \& Wilkins. Available at: http://meded.lwwhealthlibrary.com/book.aspx?boo $\mathrm{kid}=823$. Accessed May 4, 2020.

14. Harrington RA, Elkind MSV, Benjamin IJ. Protecting medical trainees on the COVID-19 frontlines saves us all. Circulation. 2020;141:e775-7.

15. DeFilippis EM, Ranard LS, Berg DD. Cardiopulmonary resuscitation during the COVID-19 pandemic: a view from trainees on the frontline. Circulation. 2020 141:1833-5.

16. Angelos P. Surgeons, ethics, and COVID-19: early lessons learned. J Am Coll Surg. 2020;230:1119-20.

17. Bates T, Cecil E, Greene I. The effect of the EWTD on training in general surgery: an analysis of electronic logbook records. Bull Roy Coll Surg Engl. 2007;89:106-9.

18. Varley I, Keir J, Fagg P. Changes in caseload and the potential impact on surgical training: a retrospective review of one hospital's experience. BMC Med Educ 2006;6:6.

19. McBride KE, Brown KGM, Fisher OM, Steffens D, Yeo DA, Koh CE. Impact of the COVID-19 pandemic on surgical services: early experiences at a nominated COVID-19 centre. ANZ J Surg. 2020;90:663-5.

20. Porpiglia F, Checcucci E, Amparore D, Verri P, Campi R, Claps F, et al. Slowdown of urology residents' learning curve during COVID-19 emergency. BJU Int. 2020;125:E15-7.

21. 100th Annual Meeting A Virtual Learning Experience. Available at: https://www aats.org/aatsimis/AATSWeb/Association/Meetings/Annual_Meeting/100th_ Annual_Meeting_Virtual/100th_Annual_Meeting_A_Virtual_Learning_Ex perience.aspx. Accessed May 4, 2020.

22. Alvin MD, George E, Deng F, Warhadpande S, Lee SI. The impact of COVID-19 on radiology trainees. Radiology. 2020;201222.

23. Covid-19 - ARCP and coding informationconference of postgraduate medical deans; 2020. Available at: https://www.copmed.org.uk/publications/covid-20. Accessed May 4, 2020

Key Words: training, residency, medical education, surgical education, COVID-19, pandemic 